\title{
More General Sudden Singularities
}

\author{
John D. Barrow \\ DAMTP, Centre for Mathematical Sciences, \\ Cambridge University, \\ Wilberforce Road, \\ Cambridge CB3 0WA, UK
}

\begin{abstract}
We present a general form for the solution of an expanding generalrelativistic Friedmann universe that encounters a singularity at finite future time. The singularity occurs in the material pressure and acceleration whilst the scale factor, expansion rate and material density remain finite and the strong energy condition holds. We also show that the same phenomenon occurs, but under different conditions, for Friedmann universes in gravity theories arising from the variation of an action that is an arbitrary analytic function of the scalar curvature.
\end{abstract}

There has been considerable interest in the possibility of finite and infinite future-time cosmological singularities in general relativity under the headings of 'big-rip' singularities and associated 'phantom matter' evolution [1] 2]. These studies have also extended to several quantum and brane-world generalisations [3], 4] [5], 6], 7] 8]. A demarcation of these and related finite-time singularities has been given in 9 .

We consider the most general form of sudden, or finite-time, singularity of the sort discussed in [10] that can arise in an Friedmann expanding universe. In 10] we showed that singularities of this sort can arise from a divergence of the pressure, $p$, at finite time despite the scale factor, $a(t)$, the material density, $\rho$, and the Hubble expansion rate, $H=\dot{a} / a$ remaining finite. The pressure singularity is accompanied by a divergence in the acceleration of the universe, $\ddot{a}$, at finite time. Remarkably, these singularities occur without violating the strong energy conditions $\rho>0$ and $\rho+3 p>0$. They can even prevent a closed Friedmann universe that obeys these energy conditions from attaining an expansion maximum [11]. In order to prevent the occurrence of these sudden singularities it is necessary to bound the pressure. A sufficient condition is to require $d p / d \rho$ to be continuous or $p / \rho$ to be finite [10.

Consider the Friedmann universe with curvature parameter $k$, and $8 \pi G=$ $c=1$, the Einstein equations reduce to 


$$
\begin{aligned}
3 H^{2} & =\rho-\frac{k}{a^{2}}, \\
0 & =\dot{\rho}+3 H(\rho+p), \\
\frac{\ddot{a}}{a} & =-\left(\frac{\rho+3 p}{6}\right) .
\end{aligned}
$$

In [10] we constructed an explicit example by seeking, over the time interval $0<t<t_{s}$, a solution for the scale factor $a(t)$ of the form

$$
a(t)=1+B t^{q}+C\left(t_{s}-t\right)^{n},
$$

where $B>0, q>0, C$ and $n>0$ are free constants to be determined. If we fix the zero of time by requiring $a(0)=0$, so $C t_{s}^{n}=-1$, we have

$$
a(t)=\left(\frac{t}{t_{s}}\right)^{q}\left(a_{s}-1\right)+1-\left(1-\frac{t}{t_{s}}\right)^{n},
$$

where $a_{s} \equiv a\left(t_{s}\right)$. Hence, as $t \rightarrow t_{s}$ from below, we have

$$
\ddot{a} \rightarrow q(q-1) B t^{q-2}-\frac{n(n-1)}{t_{s}^{2}\left(1-\frac{t}{t_{s}}\right)^{2-n}} \rightarrow-\infty
$$

whenever $1<n<2$ and $0<q \leq 1$; the solution exists on the interval $0<t<t_{s}$. Hence, as $t \rightarrow t_{s}$ we have $a \rightarrow a_{s}, H \rightarrow H_{s}$ and $\rho \rightarrow \rho_{s}>0$ where $a_{s}, H_{s}$, and $\rho_{s}$ are all finite but $p_{s} \rightarrow \infty$. By contrast, as $t \rightarrow 0$ there is an initial allencompassing strong-curvature singularity, with $H \rightarrow \infty, \rho \rightarrow \infty$ and $p \rightarrow \infty$. From (6) and (3), we see that $\rho$ and $\rho+3 p$ remain positive throughout the evolution but, because $\rho$ is finite asymptotically, the dominant-energy condition, $|p| \leq \rho$, must always be violated as $t \rightarrow t_{s}$, 10. A solution with similar properties which expands from a de Sitter past state also exists, with

$$
a(t)=a_{s}-1+\exp \left\{\lambda\left(t_{s}-t\right)\right\}-\left(1-\frac{t}{t_{s}}\right)^{n}
$$

with $\lambda>0$ constant and $1<n<2$.

The particular solution (5) expands with

$$
a(t) \approx n \frac{t}{t_{s}}+\left(\frac{t}{t_{s}}\right)^{q}\left(a_{s}-1\right) \approx\left(\frac{t}{t_{s}}\right)^{q}
$$

as $t \rightarrow 0$ and so with choices $q=1 / 3,1 / 2,2 / 3$,or $q=1$ it will resemble a massless scalar-, radiation-, dust-, or negative-curvature-dominated Friedmann universe respectively at early times. At late times, as the 'big-rip' singularity is approached, the expansion approaches a constant with

$$
a(t) \approx a_{s}+q\left(1-a_{s}\right)\left(1-\frac{t}{t_{s}}\right)
$$

as $t \rightarrow t_{s}$. 
Consider now the most general form of a solution of this type. In the neighbourhood of the singularity at $t=t_{s}$ the general formal solution of the Friedmann equations has the form

$$
a(t)=\left(t_{s}-t\right)^{n}\left[\sum_{j=0}^{\infty} \sum_{k=0}^{N_{j}} a_{j k}\left(t_{s}-t\right)^{j / Q}\left(\log ^{k}\left[t_{s}-t\right]\right)\right]
$$

where $[\ldots]$ is a convergent double Psi-series which tends to zero as $t \rightarrow t_{s} ; a_{j k}$ are constants, $N_{j} \leq j$ are positive integers and $Q \in \mathbb{Q}^{+}$(this is derived as an application of the theorem of Goriely and Hyde proved in ref. [12).

Hence, we have a generalisation of the solution (5) of the form

$$
a(t)=\left(\frac{t}{t_{s}}\right)^{q}\left(a_{s}-1\right)+1-\left(t_{s}-t\right)^{n}\left[\sum_{j=0}^{\infty} \sum_{k=0}^{N_{j}} a_{j k}\left(t_{s}-t\right)^{j / Q}\left(\log ^{k}\left[t_{s}-t\right]\right)\right] .
$$

As $t \rightarrow t_{s}$ if we choose $1<n<2,0<q<1$, we have $a \rightarrow a_{s}$ and

$$
\ddot{a} \rightarrow-n(n-1) A\left(t_{s}-t\right)^{n-2} \log ^{k}\left(t_{s}-t\right)+\text { higher order } \rightarrow-\infty,
$$

where $A>0$ is constant,and so $\rho+3 p>0$ and $p>|\rho|$ as the future singularity is approached with $p \rightarrow \infty$ at $t_{s}$. As $t \rightarrow 0$ the solution evolves as $a \approx c_{0}+c_{1} t^{q}$ and approaches the behaviour of the radiation-dominated Friedmann universe for $q=1 / 2$. As $t \rightarrow \infty$ the solution approaches $a_{s}$ in accord with eq. (91). We see that the solution (5) is the special case with all $a_{j k}=0$ except for $a_{00} \neq 0$.

By choosing $n$ to lie in the intervals $(N, N+1)$ for $N \geq 2$ where $N \in \mathbb{Z}^{+}$, we can create a singularity in which

$$
\frac{d^{N+1} a}{d t^{N+1}} \rightarrow \infty
$$

but

$$
\frac{d^{r} a}{d t^{r}} \rightarrow 0, \text { for } r \leq N \in \mathbb{Z}^{+} .
$$

In particular, this allows for singularities of the pressure which are accompanied by divergences of higher time-derivatives of $a(t)$ in Friedmann solutions of higher-order gravity theories 13. Consider a generalisation of general relativity to a theory of gravity derived from the variation of a lagrangian that is an arbitrary polynomial function $f(R)$ of the scalar curvature, $R$. The Friedmann universe is now prescribed by the solution of any two of equations (2) and the generalisations of (3) and (1), which are given respectively by [13]

$$
R f^{\prime}-2 f+3 f^{\prime \prime}\left(\ddot{R}+3 \frac{\dot{a}}{a} \dot{R}\right)+3 f^{\prime \prime \prime} \dot{R}^{2}=3 p-\rho
$$


and

$$
3 \frac{\ddot{a}}{a} f^{\prime}+\frac{1}{2} f-3 f^{\prime \prime} \dot{R} \frac{\dot{a}}{a}+\rho=0
$$

where $f^{\prime} \equiv d f / d R$ etc, and the scalar curvature of the Friedmann metric is given by

$$
R=-6\left(\frac{\ddot{a}}{a}+\frac{\dot{a}^{2}}{a^{2}}+\frac{k}{a^{2}}\right) .
$$

The equations of general relativistic cosmology in the absence of a cosmological constant arise from (15)-(17) as the special case $f(R)=R$. Various auxiliary conditions on the form of $f(R)$ are required to ensure the existence of Friedmann solutions, see ref. 13. for a discussion. We ignore them as they do not affect our results here.

In order to obtain a finite-time singularity of the sort described by (5) or (11) we need to accommodate a singularity of the pressure, $p$, in (2) in equations (15)-(16). We require that all the terms in the generalised Friedmann first integral (16) remain finite as $t \rightarrow t_{s}$ and $a \rightarrow a_{s}$. Thus we require that $\ddot{a}, f(R), f^{\prime}(R), f^{\prime \prime}(R), \dot{R}, R$ and $\rho$ are all finite in this limit.

Now consider the evolution equation (15) as $t \rightarrow t_{s}$. Since $R, f, f^{\prime}, f^{\prime \prime}$ and $f^{\prime \prime \prime}$ will be finite at $t_{s}$ we must have

$$
f^{\prime \prime} \ddot{R} \rightarrow p \rightarrow-\infty \text { as } t \rightarrow t_{s} .
$$

Hence, there must be a divergence of

$$
\ddot{R} \rightarrow-6 \frac{\dddot{a}}{a} \rightarrow-\infty \text { as } t \rightarrow t_{s}
$$

but with $R\left(t_{s}\right)$ and $\dot{R}\left(t_{s}\right)$ remaining finite. This occurs so long as $3<n<4$, since for a solution of the form (5)

$$
\lim _{t \rightarrow t_{s}} \frac{d^{r} a}{d t^{r}}=-\frac{n(n-1) . .(n-r+1)}{t_{s}^{r}}\left(1-\frac{t}{t_{s}}\right)^{n-r}
$$

and the same condition holds for the general solution (11). In the general relativity case of $f(R)=R$, the pressure singularity accompanies one in $\ddot{a}$ and requires $1<n<2$.

This generalisation of the conditions for the existence of sudden singularities to the Friedmann universes of higher-order lagrangian theories of gravity shows that the higher-order curvature corrections that these extensions introduce are able to moderate but not remove the finite-time singularity. They merely change the evolutionary behaviours of the expansion scale factor which permit their occurrence. As in general relativity, an energy condition that bounds $p / \rho$ suffices to exclude them. 


\section{References}

[1] Starobinsky A A 2000 Grav. Cosmol. 6 157; Caldwell, R R 2002 Phys. Lett. B 545 23; Shulz A and White M J 2001 Phys. Rev. D 64 043514; Hao J and Li, X 2003 Phys. Rev. D 67 107303; Gibbons G W hep-th/0302199. Nojiri S and Odintsov S D 2003 Phys. Lett. B 562147 and B 571 1; Singh P, Sami M and Dadhich N 2003 Phys. Rev. D 68 023522; Hao J and Li, X 2003 Phys. Rev. D 6804351 and 083514; Dąbrowski M, Stachowiak T and Sydlowski M 2003 Phys. Rev. D 68 067301; Elizalde P and Quiroga J 2004 Mod Phys Lett A 19 29; González-Díaz P F 2004 Phys Lett B 586 1; Feinstein A and Jhingan S 2004 Mod Phys Lett A 19 457; Chimento L P and Lazkoz R 2003 Phys Rev Lett 91 211301; Elizalde E, Nojiri S, and Odintsov S D 2004 Phys.Rev. D 70 043539; Chimento L P and Lazkoz R 2004 Mod Phys Lett A 69123512.

[2] González-Díaz P F 2003 Phys. Rev. 68 021303(R)

[3] Calcagni G 2004 Phys Rev D 69103508

[4] Gorini V, Kamenshchik A, Moschella U and Pasquier V 2004 Phys Rev D 69123512

[5] Kofinas G, Maartens R and Papantonopoulos E 2003 JHEP 0310066

[6] Sahni V and Shtanov Y 2002 Class. Quantum Grav. 19 L101

[7] Nojiri S and Odintsov S D 2004 Phys. Lett. B 5951

[8] Nojiri S and Odintsov S D Preprint hep-th/0408170

[9] Cotsakis S and Klaoudatou I Preprint gr-qc/0409022

[10] Barrow J D 2004 Class. Quantum Grav. 21 L79

[11] Barrow J D, Galloway G J and Tipler F J 1986 Mon. Not. R. astron. Soc. 223835

[12] Goriely A and Hyde C 2000 J. Diff. Eqns. 161422

[13] Barrow J D and Ottewill A C 1983 J. Phys. A 162757 